\title{
Transcatheter aortic valve replacement; a kidney's perspective
}

\author{
Wisit Cheungpasitporn $^{1}$, Charat Thongprayoon ${ }^{2}$, Kianoush Kashani ${ }^{3 *}$ \\ ${ }^{1}$ Division of Nephrology and Hypertension, Department of Internal Medicine, Mayo Clinic, Rochester, MN, USA \\ ${ }^{2}$ Department of Internal Medicine, Bassett Medical Center, Cooperstown, NY, USA \\ ${ }^{3}$ Division of Pulmonary and Critical Care Medicine, Department of Internal Medicine, Mayo Clinic, Rochester, MN, USA
}

\section{A R T I C L E I N F O}

\section{Article Type:}

Review

\section{Article History:}

Received: 11 October 2015

Accepted: 5 January 2016

Published online: 18 January 2016

\section{Keywords:}

Acute kidney injury

Chronic kidney disease

Dialysis

Transcatheter aortic valve implantation

Transcatheter aortic valve replace-

ment

Transplantation

\begin{abstract}
A B S T R A C T
Transcatheter aortic valve replacement (TAVR) has now emerged as a viable treatment option for high-risk patients with severe aortic stenosis (AS) who are not suitable candidates for surgical aortic valve replacement (SAVR). Despite encouraging published outcomes, acute kidney injury (AKI) is common and lowers the survival of patients after TAVR. The pathogenesis of AKI after TAVR is multifactorial including TAVR specific factors such as the use of contrast agents, hypotension during rapid pacing, and embolization; preventive measures may include pre-procedural hydration, limitation of contrast dye exposure, and avoidance of intraprocedural hypotension. In recent years, the number of TAVR performed worldwide has been increasing, as well as published data on renal perspectives of TAVR including AKI, chronic kidney disease, end-stage kidney disease, and kidney transplantation. This review aims to present the current literature on the nephrology aspects of TAVR, ultimately to improve the patients' quality of care and outcomes.
\end{abstract}

Implication for health policy/practice/research/medical education:

Transcatheter aortic valve replacement (TAVR) has now emerged as a viable treatment option for high-risk patients with severe aortic stenosis (AS) who are not suitable candidates for surgical aortic valve replacement (SAVR). Despite encouraging published outcomes, acute kidney injury (AKI) after TAVR is common and lowers the survival of patients after TAVR. In recent years, the number of TAVR performed worldwide have been increasing as well as published data on renal perspectives of TAVR including AKI, chronic kidney disease, end-stage kidney disease and kidney transplantation. We present the current literature on nephrology aspects of TAVR, ultimately to improve the patients' quality of care and outcomes.

Please cite this paper as: Cheungpasitporn W, Thongprayoon C, Kashani K. Transcatheter aortic valve replacement; a kidney's perspective. J Renal Inj Prev. 2016;5(1):1-7. DOI: 10.15171/jrip.2016.01

\section{Introduction}

Aortic stenosis (AS) is one of the most common cardiac degenerative valvular diseases, with a prevalence of $1.3 \%$ in patients between 65 and 74 years and $2.8 \%-4.6 \%$ in patients $>75$ years of age (1-3). Due to an aging population, the incidence of AS continues to rise over time, and thus, AS has become a significant healthcare burden $(1,3,4)$. Without treatment, these patients have a poor prognosis with 50\% mortality in the first two years after diagnosis (5).
Surgical aortic valve replacement (SAVR) is currently considered the gold standard treatment for severe symptomatic AS (6). Transcatheter aortic valve replacement (TAVR), also known as transcatheter aortic valve implantation (TAVI), being performed since 2002, has now emerged as a viable treatment option for high-risk patients with severe AS who are not suitable candidates for SAVR (6-9). Recently published results of the 5-year outcomes from multicenter, randomized controlled trials (RCTs) 
demonstrated a survival benefit of TAVR over standard treatment for patients with inoperable AS (10) and comparable survival rates in high-risk patients with AS undergoing TAVR compared to SAVR (11). The applications of TAVR are also expanding to 'off-label' indications in patients with intermediate risk, AS secondary to bicuspid valve disease, aortic regurgitation, aortic valve-in-valve procedures, and mitral valve interventions (12). To date over 200000 procedures have been performed worldwide. Despite the encouraging reports, post-procedural acute kidney injury (AKI) remains a common complication of TAVR, particularly when carried out in patients with high comorbidities $(13,14)$.

\section{Materials and methods}

This review article discusses kidney related aspects of TAVR including the incidence, predictors, and the impact of AKI following TAVR. We report the available evidence of clinical outcomes of patients with chronic kidney disease (CKD), end-stage renal disease (ESRD), and renal transplantation who undergo TAVR.

For this review, we used a variety of sources by searching through PubMed, EMBASE, Scopus and directory of open access journals (DOAJ). The search was performed using combinations of the following key words and or their equivalents; acute kidney injury, chronic kidney disease, dialysis, transcatheter aortic valve implantation, transcatheter aortic valve replacement and transplantation.

\section{AKI after TAVR}

\section{Incidence of AKI After TAVR}

Due to many different definitions of AKI used in the literature $(15,16)$, the reported incidence of AKI after TAVR varies widely $(13,14)$. By using a consensus AKI definition (modified RIFLE) (17), the reported incidence of AKI following TAVR ranged from $15 \%$ to $57 \%$, with the need for renal replacement therapy (RRT) in $2 \%-40 \%$ of all patients $(6,13,14,18-24)$. Compared to patients without AKI, patients who developed AKI after TAVR had a higher mortality rate of $9 \%-44 \%$ at 30 days and $32 \%-56 \%$ at 1 year $(13,14)$.

In 2012, the Valve Academic Research Consortium (VARC) published their updated endpoint definitions in the VARC-2 consensus (25) recommending standardized criteria, i.e., the Acute Kidney Injury Network (AKIN) criteria, and most recently, the Kidney Disease Improving Global Outcomes (KDIGO) criteria (26). In addition,
VARC-2 standardized the timing for the AKI diagnosis, extending from 72 hours to 7 days following a TAVR procedure. With these standardized criteria, we recently reported the incidence of AKI within 7 days following TAVR of $28 \%$ ( $22 \%$ in stage $1,2 \%$ in stage 2 , and $4 \%$ in stage 3 ) and the need for RRT during hospitalization of $3 \%$ (27).

\section{Pathophysiology and risk factors of AKI after TAVR}

Similar to SAVR-associated AKI, the pathogenesis of TAVR-related AKI is multifactorial including perioperative renal hypoperfusion related to a combination of pre-, intra- and postoperative factors (Table 1) $(13,14,28)$. Although the use of cardiopulmonary bypass during TAVR is not required, TAVR itself is associated with specific AKI risks. Catheter-based techniques and valve implantation, use of contrast agents, hypotension during rapid ventricular pacing for balloon valvuloplasty and valve deployment, and embolization resulting from the manipulation of catheters in the aorta of patients with diffuse atherosclerosis are examples of intraoperative risk factors for AKI (28).

\section{Transapical approach and cholesterol emboli}

The TAVR procedure can be performed through a few approaches such as transfemoral (TF), transapical (TA) and transaortic routes. There are a few advantages to using TFTAVR over other techniques, including the ability to use moderate sedation and local anesthetics and shorter procedure and recovery times (29). Therefore, at most centers the TF-TAVR strategy is considered first $(13,14,28,29)$. However, not all patients are suitable for TF-TAVR due to advanced peripheral vascular disease; in these cases, very small or severely calcified or tortuous peripheral vessels can preclude safe placement of the access sheath $(28,29)$. For these patients, TA-TAVR is the method of choice (30). Unfortunately, several studies have demonstrated an association between the TA approach and higher risk of AKI (31-33). The mechanisms of observed higher AKI risk are only speculative, but a possible explanation could be the difference in patient populations. Those undergoing a TA-TAVR have more severe peripheral vascular atherosclerotic disease, which is per se a risk factor for AKI after TAVR (Table 1) $(34,35)$. In addition, the aortas of patients with severe peripheral vascular disease are usually more atherosclerotic. During the TA-TAVR procedure, the instrumentation of the aorta may result in the dislodgement of calcium plaques and cholesterol emboli to the renal vascular bed, leading to AKI (35). Also, as discussed earlier,

Table 1. Reported potential predictors/perioperative factors associated with postoperative AKI following TAVR ${ }^{a}$

\begin{tabular}{lll}
\hline Preoperative & Intraoperative & Postoperative \\
\hline -Older age & -Periprocedural Bleeding and Blood Transfusion & -Vasoconstricting agents \\
-Pre-existing chronic kidney disease & -Embolic events & Nephrotoxins \\
-Short-interval contrast exposure & -Contrast agents & -Decreased heart function \\
-Congestive heart failure & -Hypotension from rapid ventricular pacing & -Hemodynamic instability \\
-Peripheral Vascular Disease & -Transapical approach & -Grade of aortic regurgitation after the \\
-Diabetes & -Complicated cases requiring intra-aortic balloon & procedure \\
-Logistic EuroSCORE & pump & \\
\hline
\end{tabular}

Abbreviations: AKI, acute kidney injury; TAVR, Transcatheter Aortic Valve Replacement.

${ }^{a}$ References 13, 14, 28, and 35. 
TA-TAVR is mostly performed under general anesthesia, which could be associated with general and renal hypoperfusion, while TF-TAVR is performed under moderate sedation and local anesthetics and is commonly not associated with hemodynamic instability (29).

\section{Contrast agent exposure}

Despite advances in TAVR technique, the TAVR procedure still requires fluoroscopy and angiography using contrast agent to aid in positioning the valve (36), which may result in contrast-induced AKI (CIAKI). However, the impact of contrast agent utilization on AKI after TAVR remains controversial. A few studies suggest an association between contrast media and higher AKI incidence following TAVR $(37,38)$, especially in patients with preexisting CKD (39). However, other reports have not demonstrated such association (32,34,40-43). Minimization of the contrast dose during TAVR to $<100 \mathrm{~mL}$ and use of low or iso-osmolar contrast media can explain these observations $(34,35,37,44)$.

\section{Hypotension from rapid ventricular pacing}

During the TAVR procedure, the positioning of balloonexpandable valves requires rapid ventricular pacing via a right ventricular temporary pacing wire to reduce aortic pressure and achieve cardiac standstill $(14,29)$. It has been suggested that excessive hypotension, induced by rapid ventricular pacing, may theoretically lead to a decrease in renal perfusion and renal ischemia-reperfusion injury, with an increased risk of AKI. Interestingly, Bagur et al (41) evaluated the number of procedural rapid pacing runs in the development of AKI following TAVR and found no significant correlation. Despite this report, the impact of the rapid pacing duration on AKI following TAVR requires more detailed investigation $(35,41)$.

AKI after transcatheter or surgical aortic valve replacement Among studies that compared the incidence of postoperative AKI events in patients with severe AS undergoing TAVR versus SAVR (13), several studies showed a higher incidence of AKI among patients who underwent TAVR $(23,45)$. However, it should be noted that patients selected for TAVR typically have higher comorbidities which may carry a higher risk for AKI. Therefore, we recently conducted a meta-analysis of 3 randomized control trials with a total of 1852 patients and 14 cohort studies with 3113 patients and found a lower AKI risk among TAVR patients when compared with SAVR (13).

It is unclear whether the different study outcomes were due to patient risk profiles confounding the results, or whether the heterogeneity of AKI definitions among included studies were the cause. Thus, we undertook a study of 1563 adult patients undergoing isolated TAVR or SAVR for severe AS at Mayo Clinic Hospital in Rochester, Minnesota from January 1, 2008, to June 30, 2014 (27). We performed a propensity-matched comparison for the postoperative incidence of KDIGO-defined AKI within 7 days of the procedure as recommended by the VARC-2 consensus (25). Among the 195 matched pairs (390 patients), baseline characteristics, including Society of Thoracic Surgeons (STS) risk score and estimated glomerular filtration rate eGFR, were comparable between the two groups. We found no significant difference in postoperative AKI incidence (24.1\% versus $29.7 \% ; P=0.21$ ) between the TAVR and SAVR groups. In addition, there were no differences in major adverse kidney events, the composite of in-hospital death, use of RRT during hospitalization and persistence of renal dysfunction at hospital discharge ( $2.1 \%$ versus $1.5 \% ; P=0.70)$, or mortality $>6$ months after surgery (6.0\% versus $8.3 \%$; $P=0.51)(27)$. Thus, TAVR did not affect postoperative AKI risk and may be preferred in high-risk patients with severe AS considering its less invasive nature compared to SAVR.

\section{TAVR in CKD}

Based on the data from the Edwards SAPIEN Aortic Bioprosthesis European Outcome (SOURCE) Registry, CKD is considered one of the strongest independent predictors of 1-year mortality following TAVR (46). It is well established that patients with CKD carry a higher risk of AKI (47). Interestingly, in the setting of AKI after TAVR, studies have demonstrated different findings regarding the impact of CKD on AKI occurrence. Elhmidi et al (40) studied 234 patients with severe AS who underwent TAVR between 2007 and 2010 at a single center and found that preoperative serum creatinine level was the only independent predictor of postoperative AKI. Khawaja et al (48), subsequently demonstrated that higher CKD stage had the strongest independent associations with AKI after TAVR. However, a number of studies did not detect an association between CKD and AKI following TAVR $(31,34,41,49)$.

Voigtländer et al (50), recently studied the influence of kidney function before TAVR on the AKI incidence in 540 patients. Investigators divided patients into three groups according to their GFR before TAVR (GFR $\geq 60$ (normal renal function), 30-59 (moderate impaired renal function), and $<30 \mathrm{~mL} / \mathrm{min} / 1.73 \mathrm{~m}^{2}$ [severe impaired renal function]). Overall, there was an increase in GFR after TAVR from baseline GFR of $59.1 \pm 21.7 \mathrm{ml} / \mathrm{min} / 1.73 \mathrm{~m}^{2}$ to $63.6 \pm 23.6 \mathrm{ml} / \mathrm{min} / 1.73 \mathrm{~m}^{2}$ at hospital discharge. The investigators demonstrated a modest increase in GFR in the moderately impaired renal function group and a significant increase in GFR in those with severe decreased renal function. There was no significant change in GFR after TAVR in patients with normal renal function (50). The improvement of GFR was also demonstrated at one month following TAVR in patients with preexisting CKD (51). It is therefore possible that renal function improved in some cases following TAVR due to the improvement of cardiac performance following correction of valvular disease (28).

\section{TAVR in ESRD}

Valvular heart disease, and especially AS, is more prevalent in patients with ESRD undergoing maintenance dial- 
ysis than the general population due to calcification of the aortic valve associated with secondary hyperparathyroidism (52). In ESRD patients, aortic valve calcification occurs 10-20 years earlier and progresses more rapidly than in the general population (53). In addition, ESRD patients often carry multiple comorbidities and bear a high risk of complications following SAVR. On the other hand, the mortality rate is higher and quality of life in lower when ESRD patients have symptomatic AS (53). Recently, Kobrin et al (54) studied all Medicare fee-for-service patients (5005 undergoing TAVR and 32634 undergoing SAVR) between January 1, 2011, and November 30, 2012. Compared to non-dialysis patients, TAVR patients on dialysis had a significantly higher rate of mortality at 30 days (13\% vs. $6 \%$ ) and lower survival at one year (57.4\% vs. $77.4 \%)$. In the propensity-matched comparison of 194 matched pairs of dialysis SAVR and dialysis TAVR patients (388 patients), the investigators reported shorter length of hospital stay with comparable survival in TAVR patients receiving dialysis (54). Thus, TAVR potentially plays a significant role in patients with ESRD and severe AS.

\section{TAVR in kidney transplant recipients}

During kidney transplant candidate evaluation, screening for AS with history, physical examination, and echocardiogram is recommended for all patients if clinical suspicion for AS is high $(55,56)$. It is recommended that patients with moderate to severe AS be considered for valve replacement before kidney transplantation. Even after transplantation, cardiovascular and valvular diseases are still prevalent and remain one of the leading causes of death in kidney transplant recipients (57). Furthermore, the incidence of AS among kidney transplant patients will likely rise as their survival improves and as the mean age of patients undergoing kidney transplantation increases (58). Data on the survival of kidney transplantation patients after cardiac valve replacement are limited. Recent data from the US Renal Data System database demonstrated mortality rates of kidney transplant recipients undergoing valvular heart surgery of $14 \%$ in the hospital and $40 \%$ within 2 years of surgery (57). In addition, kidney transplant recipients with severe AS are often found unsuitable for SAVR due to impaired kidney function, possible side effects of immunosuppressive medication, and comorbidities (58).

Fox et al (58) reviewed the outcomes of eight kidney transplant recipients with severe AS undergoing TAVR (6 transfemoral; 2 transapical). The investigators reported that all TAVR procedures were performed successfully with excellent functional results. After TAVR, all kidney transplant recipients were alive at the 12-month followup with only one reported cardiovascular event. Despite encouraging outcomes after TAVR in kidney transplant recipients, aortic root rupture, a rare but fatal complication of TAVR, was recently reported in two renal transplant patients (59). However, it is still unclear if chronic immunosuppressant therapy is associated with aortic root rupture, and future studies are needed for transplant re- cipients undergoing TAVR.

\section{Measures to prevent AKI after TAVR}

As discussed earlier in this review, the pathogenesis of AKI after TAVR is multifactorial. Measures to prevent AKI after TAVR are proposed in Table 2. Although the data on the impact of contrast exposure on the incidence of AKI after TAVR are still controversial, avoiding repeated exposure to contrast dye over a short period, minimizing the volume of contrast agent, especially in patients with $\mathrm{CKD}$, and careful hydration based on the individual's cardiac performance should be considered. Intraprocedural hypotension should be avoided in all cases. Patients who undergo TAVR with a transapical approach, patients with renal insufficiency, or patients requiring an intra-aortic balloon pump use should be considered at risk for developing AKI after TAVR, and preventive measures including preprocedural hydration, limitation of potentially nephrotoxic agents, and a judicious use of blood transfusions should be considered.

The RenalGuard system, a dedicated device with forced diuresis and matched hydration designed for CIAKI, has recently been introduced in the TAVR setting and appears to be safe and efficient (60). Although investigators demonstrated a smaller creatinine rise in a RenalGaurd group compared to a standard of care cohort, they did not report differences in important clinical endpoints, including rate of RRT or mortality $(60,61)$. While the attempts to reduce stroke rates by TAVR embolic protection systems are promising (62), future studies on intervention or preventive measures to prevent renal emboli, especially in patients with $\mathrm{CKD}$, are needed. Another intervention which requires further validation is the use of a Doppler-based renal resistance index to predict and identify patients at risk for AKI (63). In the future, progress in the research of urine biomarkers (64), electronic health records with an AKI alert (65) for early AKI detection, and risk stratification models will likely improve the renal outcomes of patients undergoing TAVR.

\section{Conclusion}

The prevalence of AS continues to rise over time among our aging population. With advances in the field of interventional cardiology, TAVR has already become a treatment option for severe AS in the inoperable or high-risk

Table 2. Measures to prevent acute kidney injury after transcatheter aortic valve replacement

\footnotetext{
General measures

Minimize repeated exposure to contrast dye during a short period

Minimize the volume of contrast agent

Avoid intraprocedural hypotension

Avoid bleeding and restrict the use of blood transfusions

Potential future preventive measures

RenalGuard system (Forced diuresis with matched hydration)

Doppler-based renal resistance index top predict risk for AKI

TAVR Embolic Protection Systems to prevent renal embolism

Abbreviations: AKI, acute kidney injury; TAVR, transcatheter aortic valve replacement.
} 
surgical candidate. In addition, the use of TAVR for the treatment of other pathologies and lower-risk patients is being explored. The number of TAVR procedures will continue to increase in the general patient population as well as in patients with CKD, ESRD, and in candidates for kidney transplantation. Thus, physicians should understand and be aware of potential complications following the TAVR procedure. The occurrence of AKI following TAVR is common and a prognostically significant complication. The ultimate success of TAVR depends on careful attention to detail and prompt management of complications and understanding the risk factors for AKI after TAVR. Growing knowledge of the potential impact of TAVR on kidney function will help improve patient selection, TAVR technique, and preventive measures to improve patients' outcomes.

\section{Authors' contribution}

WC, CT, and KK contributed to the manuscript equally.

\section{Conflicts of interest}

The authors declare that they have no conflicting interest.

\section{Ethical considerations}

Ethical issues (including plagiarism, data fabrication, double publication) have been completely observed by the authors.

\section{Funding/Support}

None.

\section{References}

1. Lung B, Vahanian A. Epidemiology of acquired valvular heart disease. Can J Cardiol. 2014;30:962-70.

2. Nkomo VT, Gardin JM, Skelton TN, Gottdiener JS, Scott CG, Enriquez-Sarano M. Burden of valvular heart diseases: A population-based study. Lancet. 2006;368:1005-11.

3. Eveborn GW, Schirmer H, Heggelund G, Lunde P, Rasmussen $\mathrm{K}$. The evolving epidemiology of valvular aortic stenosis. The Tromso study. Heart. 2013;99:396-400.

4. Mangieri A, Regazzoli D, Ruparelia N, Colombo A, Latib A. Recent advances in transcatheter aortic valve replacement for high-risk patients. Expert Rev Cardiovasc Ther. 2015;13:1237-49.

5. Brown JM, O'Brien SM, Wu C, Sikora JA, Griffith BP, Gammie JS. Isolated aortic valve replacement in North America comprising 108,687 patients in 10 years: changes in risks, valve types, and outcomes in the Society of Thoracic Surgeons National Database. J Thorac Cardiovasc Surg. 2009;137:82-90.

6. Adams DH, Popma JJ, Reardon MJ, Yakubov SJ, Coselli JS, Deeb GM, et al. Transcatheter aortic-valve replacement with a self-expanding prosthesis. N Engl J Med. 2014;370:17908.

7. O'Brien SM, Shahian DM, Filardo G, Ferraris VA, Haan CK, Rich JB, et al. The Society of Thoracic Surgeons 2008 cardiac surgery risk models: Part 2--isolated valve surgery. Ann Thorac Surg. 2009;88:S23-42.

8. Nishimura RA, Otto CM, Bonow RO, Carabello BA, Erwin JP 3rd, Guyton RA, et al. 2014 AHA/ACC Guideline for the Management of Patients With Valvular Heart Disease: A report of the American College of Cardiology/American Heart Association Task Force on Practice Guidelines. Circulation. 2014;129:e521-643.

9. Reardon MJ, Adams DH, Kleiman NS, Yakubov SJ, Coselli JS, Deeb GM, et al. 2-year outcomes in patients undergoing surgical or self-expanding transcatheter aortic valve replacement. J Am Coll Cardiol. 2015;66:113-21.

10. Kapadia SR, Leon MB, Makkar RR, Tuzcu EM, Svensson LG, Kodali S, et al. 5-year outcomes of transcatheter aortic valve replacement compared with standard treatment for patients with inoperable aortic stenosis (PARTNER 1): a randomised controlled trial. Lancet. 2015;385:2485-91.

11. Mack MJ, Leon MB, Smith CR, Miller DC, Moses JW, Tuzcu $\mathrm{EM}$, et al. 5-year outcomes of transcatheter aortic valve replacement or surgical aortic valve replacement for high surgical risk patients with aortic stenosis (PARTNER 1): A randomised controlled trial. Lancet. 2015;385:2477-84.

12. Ruparelia N, Prendergast BD. TAVI in 2015: who, where and how? Heart. 2015;101:1422-31.

13. Thongprayoon C, Cheungpasitporn W, Srivali N, Ungprasert P, Kittanamongkolchai W, Greason KL, et al. Acute kidney injury after transcatheter aortic valve replacement: A systematic review and meta-analysis. Am J Nephrol. 2015;41:372-82.

14. Elhmidi Y, Bleiziffer S, Deutsch MA, Krane M, Mazzitelli $\mathrm{D}$, Lange $\mathrm{R}$, et al. Acute kidney injury after transcatheter aortic valve implantation: incidence, predictors and impact on mortality. Arch Cardiovasc Dis. 2014;107:133-9.

15. Thongprayoon C, Cheungpasitporn W, Akhoundi A, Ahmed AH, Kashani KB. Actual versus ideal body weight for acute kidney injury diagnosis and classification in critically ill patients. BMC Nephrol. 2014;15:176.

16. Thongprayoon C, Cheungpasitporn W, Kittanamongkolchai W, Srivali N, Ungprasert P, Kashani K. Optimum methodology for estimating baseline serum creatinine for the acute kidney injury classification. Nephrology. 2015;20:881-6.

17. Ricci Z, Cruz D, Ronco C. The RIFLE criteria and mortality in acute kidney injury: a systematic review. Kidney Int. 2008;73:538-46.

18. Stortecky S, Brinks H, Wenaweser P, Huber C, Pilgrim T, Windecker S, et al. Transcatheter aortic valve implantation or surgical aortic valve replacement as redo procedure after prior coronary artery bypass grafting. Ann Thorac Surg. 2011;92:1324-30.

19. Wenaweser P, Pilgrim T, Guerios E, Stortecky S, Huber C, Khattab AA, et al. Impact of coronary artery disease and percutaneous coronary intervention on outcomes in patients with severe aortic stenosis undergoing transcatheter aortic valve implantation. EuroIntervention. 2011;7:541-8.

20. Appel CF, Hultkvist H, Nylander E, Ahn H, Nielsen NE, Freter W, et al. Transcatheter versus surgical treatment for aortic stenosis: Patient selection and early outcome. Scand Cardiovasc J. 2012;46:301-7.

21. Latib A, Maisano F, Bertoldi L, Giacomini A, Shannon J, Cioni $\mathrm{M}$, et al. Transcatheter vs surgical aortic valve replacement in intermediate-surgical-risk patients with aortic stenosis: A propensity score-matched case-control study. Am Heart J. 2012;164:910-7.

22. Fusari M, Bona V, Muratori M, Salvi L, Salis S, Tamborini G, et al. Transcatheter vs. Surgical aortic valve replacement: A retrospective analysis assessing clinical effectiveness and 
safety. J Cardiovasc Med (Hagerstown). 2012;13:229-41.

23. Strauch JT, Scherner M, Haldenwang PL, Madershahian N, Pfister R, Kuhn EW, et al. Transapical minimally invasive aortic valve implantation and conventional aortic valve replacement in octogenarians. Thorac Cardiovasc Surg. 2012;60:335-42

24. Onorati F, D’Errigo P, Grossi C, Barbanti M, Ranucci M, Covello DR, et al. Effect of severe left ventricular systolic dysfunction on hospital outcome after transcatheter aortic valve implantation or surgical aortic valve replacement: Results from a propensity-matched population of the italian observant multicenter study. J Thorac Cardiovasc Surg. 2014;147:568-75.

25. Kappetein AP, Head SJ, Genereux P, Piazza N, van Mieghem $\mathrm{NM}$, Blackstone EH, et al. Updated standardized endpoint definitions for transcatheter aortic valve implantation: The Valve Academic Research Consortium-2 consensus document. Eur Heart J. 2012;33:2403-18.

26. KDIGO AKI Work Group. KDIGO clinical practice guidelines for acute kidney injury. Kidney Int. 2012;2:1138.

27. Thongprayoon C, Cheungpasitporn W, Srivali N, Harrison AM, Gunderson TM, Kittanamongkolchai W, et al. AKI after transcatheter or surgical aortic valve replacement. J Am Soc Nephrol. 2015 Oct 20. pii: ASN.2015050577

28. Scherner M, Wahlers T. Acute kidney injury after transcatheter aortic valve implantation. J Thorac Dis. 2015;7:1527-35.

29. Ruparelia N, Prendergast BD. Transcatheter aortic valve implantation - what the general physician needs to know. Clin Med (Lond). 2015;15:420-5.

30. Hamm CW, Arsalan M, Mack MJ. The future of transcatheter aortic valve implantation. JThoracDis. 2015;7:1543-7.

31. Kong WY, Yong G, Irish A. Incidence, risk factors and prognosis of acute kidney injury after transcatheter aortic valve implantation. Nephrology. 2012;17:445-451.

32. Barbash IM, Ben-Dor I, Dvir D, Maluenda G, Xue Z, Torguson R, et al. Incidence and predictors of acute kidney injury after transcatheter aortic valve replacement. Am Heart J. 2012;163:1031-6.

33. Saia F, Ciuca C, Taglieri N, Marrozzini C, Savini C, Bordoni $\mathrm{B}$, et al. Acute kidney injury following transcatheter aortic valve implantation: Incidence, predictors and clinical outcome. Int J Cardiol. 2013;168:1034-40.

34. Aregger F, Wenaweser P, Hellige GJ, Kadner A, Carrel $\mathrm{T}$, Windecker $\mathrm{S}$, et al. Risk of acute kidney injury in patients with severe aortic valve stenosis undergoing transcatheter valve replacement. Nephrol Dial Transplant. 2009;24(7):2175-9.

35. Najjar M, Salna M, George I. Acute kidney injury after aortic valve replacement: Incidence, risk factors and outcomes. Expert Rev Cardiovasc Ther. 2015;13:301-16.

36. Lichtenstein SV, Cheung A, Ye J, Thompson CR, Carere RG, Pasupati S, et al. Transapical transcatheter aortic valve implantation in humans: initial clinical experience. Circulation. 2006;114:591-6.

37. Yamamoto M, Hayashida K, Mouillet G, Chevalier B, Meguro K, Watanabe Y, et al. Renal function-based contrast dosing predicts acute kidney injury following transcatheter aortic valve implantation. JACC Cardiovasc Interv. 2013;6:479-86.

38. Van Linden A, Kempfert J, Rastan AJ, Holzhey D, Blumenstein J, Schuler G, et al. Risk of acute kidney injury after minimally invasive transapical aortic valve implantation in 270 patients. Eur J Cardiothorac Surg. 2011;39:835-42.

39. Madershahian N, Scherner M, Liakopoulos O, Rahmanian P, Kuhn E, Hellmich M, et al. Renal impairment and transapical aortic valve implantation: impact of contrast medium dose on kidney function and survival. Eur J Cardiothorac Surg. 2012;41(6):1225-32.

40. Elhmidi Y, Bleiziffer S, Piazza N, Hutter A, Opitz A, Hettich I, et al. Incidence and predictors of acute kidney injury in patients undergoing transcatheter aortic valve implantation. Am Heart J. 2011;161:735-9.

41. Bagur R, Webb JG, Nietlispach F, Dumont E, De Larochelliere R, Doyle D, et al. Acute kidney injury following transcatheter aortic valve implantation: predictive factors, prognostic value, and comparison with surgical aortic valve replacement. Eur Heart J. 2010;31:865-74.

42. Nuis RJ, Van Mieghem NM, Tzikas A, Piazza N, Otten AM, Cheng J, et al. Frequency, determinants, and prognostic effects of acute kidney injury and red blood cell transfusion in patients undergoing transcatheter aortic valve implantation. Catheter Cardiovasc Interv. 2011;77:881-9.

43. Sinning JM, Ghanem A, Steinhauser H, Adenauer V, Hammerstingl C, Nickenig G, et al. Renal function as predictor of mortality in patients after percutaneous transcatheter aortic valve implantation. JACC Cardiovasc Interv. 2010;3:1141-9.

44. Cheungpasitporn W, Thongprayoon C, Kittanamongkolchai W, Edmonds PJ, O'Corragain OA, Srivali N, et al. Periprocedural effects of statins on the incidence of contrast-induced acute kidney injury: a systematic review and meta-analysis of randomized controlled trials. Ren Fail. 2015;37:664-71.

45. Holzhey DM, Shi W, Rastan A, Borger MA, Hansig M, Mohr FW. Transapical versus conventional aortic valve replacement--a propensity-matched comparison. Heart Surg Forum. 2012;15:E4-8.

46. Thomas M, Schymik G, Walther T, Himbert D, Lefevre $\mathrm{T}$, Treede $\mathrm{H}$, et al. One-year outcomes of cohort 1 in the Edwards SAPIEN Aortic Bioprosthesis European Outcome (SOURCE) registry: The European registry of transcatheter aortic valve implantation using the Edwards SAPIEN valve. Circulation. 2011;124:425-33.

47. Singh P, Rifkin DE, Blantz RC. Chronic kidney disease: An inherent risk factor for acute kidney injury? Clin J Am Soc Nephrol. 2010;5:1690-5.

48. Khawaja MZ, Thomas M, Joshi A, Asrress KN, Wilson K, Bolter $\mathrm{K}$, et al. The effects of varc-defined acute kidney injury after transcatheter aortic valve implantation (TAVI) using the edwards bioprosthesis. EuroIntervention. 2012;8:563-70.

49. Wessely M, Rau S, Lange P, Kehl K, Renz V, Schonermarck $\mathrm{U}$, et al. Chronic kidney disease is not associated with a higher risk for mortality or acute kidney injury in transcatheter aortic valve implantation. Nephrol Dial Transplant. 2012;27:3502-8.

50. Voigtländer L, Schewel J, Martin J, Schewel D, Frerker C, Wohlmuth P, et al. Impact of kidney function on mortality after transcatheter valve implantation in patients with severe aortic valvular stenosis. Int J Cardiol. 2015;178:27581.

51. Keles T, Ayhan H, Durmaz T, Sari C, Aslan AN, Erdogan $\mathrm{KE}$, et al. Improvement in renal functions with transcatheter 
aortic valve implantation. J Geriatr Cardiol. 2013;10:31722.

52. Kajbaf S, Veinot JP, Ha A, Zimmerman D. Comparison of surgically removed cardiac valves of patients with ESRD with those of the general population. Am J Kidney Dis. 2005;46:86-93.

53. Hamilton P, Coverdale A, Edwards C, Ormiston J, Stewart J, Webster M, et al. Transcatheter aortic valve implantation in end-stage renal disease. Clin Kidney J. 2012;5:247-9.

54. Kobrin DM, McCarthy FH, Herrmann HC, Anwaruddin S, Kobrin S, Szeto WY, et al. Transcatheter and surgical aortic valve replacement in dialysis patients: A propensitymatched comparison. Ann Thorac Surg. 2015;100:1230-7.

55. Knoll G, Cockfield S, Blydt-Hansen T, Baran D, Kiberd B, Landsberg D, et al. Canadian Society of Transplantation: Consensus guidelines on eligibility for kidney transplantation. CMAJ. 2005;173:S1-25.

56. Gaston RS, Danovitch GM, Adams PL, Wynn JJ, Merion $\mathrm{RM}$, Deierhoi MH, et al. The report of a national conference on the wait list for kidney transplantation. Am J Transplant. 2003;3:775-85.

57. Sharma A, Gilbertson DT, Herzog CA. Survival of kidney transplantation patients in the united states after cardiac valve replacement. Circulation. 2010;121:2733-9.

58. Fox H, Buttner S, Hemmann K, Asbe-Vollkopf A, Doss M, Beiras-Fernandez A, et al. Transcatheter aortic valve implantation improves outcome compared to open-heart surgery in kidney transplant recipients requiring aortic valve replacement. J Cardiol. 2013;61:423-7.

59. Holper EM, Kim RJ, Brooks A, Mack M. Aortic root rupture after TAVR in two renal transplant patients on chronic immunosuppressant therapy. Catheter Cardiovasc Interv. 2015;85:909-15.

60. Barbanti M, Gulino S, Capranzano P, Imme S, Sgroi
C, Tamburino C, et al. Acute kidney injury with the RenalGuard system in patients undergoing transcatheter aortic valve replacement: The PROTECT-TAVI trial (PROphylactic effect of furosEmide-induced diuresis with matched isotonic intravenous hydraTion in Transcatheter Aortic Valve Implantation). JACC Cardiovasc Interv. 2015;8(12):1595-1604.

61. Rihal CS, Kashani KB. Prevention of acute kidney injury with the RenalGuard system in patients undergoing transcatheter aortic valve replacement: the PROTECTTAVI trial (PROphylactic effecT of furosEmide-induCed diuresis with matched isotonic intravenous hydraTion in Transcatheter Aortic Valve Implantation). JACC Cardiovasc Interv. 2015;8:1605-7.

62. Rodes-Cabau J, Kahlert P, Neumann FJ, Schymik G, Webb JG, Amarenco P, et al. Feasibility and exploratory efficacy evaluation of the Embrella Embolic Deflector system for the prevention of cerebral emboli in patients undergoing transcatheter aortic valve replacement: The PROTAVI-C pilot study. JACC Cardiovasc Interv. 2014;7:1146-55.

63. Sinning JM, Adenauer V, Scheer AC, Lema Cachiguango SJ, Ghanem A, Hammerstingl C, et al. Doppler-based renal resistance index for the detection of acute kidney injury and the non-invasive evaluation of paravalvular aortic regurgitation after transcatheter aortic valve implantation. EuroIntervention. 2014;9:1309-16.

64. Lieske JC, Chawla L, Kashani K, Kellum JA, Koyner JL, Mehta RL. Biomarkers for acute kidney injury: Where are we today? Where should we go? Clin Chem. 2014;60:294300 .

65. Kashani K, Herasevich V. Sniffing out acute kidney injury in the ICU: Do we have the tools? Curr Opin Crit Care. 2013;19:531-6.

Copyright $\odot 2016$ The Author(s); Published by Nickan Research Institute. This is an open-access article distributed under the terms of the Creative Commons Attribution License (http://creativecommons.org/licenses/by/4.0), which permits unrestricted use, distribution, and reproduction in any medium, provided the original work is properly cited. 